\title{
Rapid Fractionation and Characterisation of Alpha-Synuclein Oligomers in Solution
}

\author{
William E. Arter ${ }^{1,2, \dagger}$, Catherine K. Xu ${ }^{1, \dagger}$, Marta Castellana-Cruz ${ }^{1}$, \\ Therese W. Herling ${ }^{1}$, Georg Krainer ${ }^{1}$, Kadi L. Saar ${ }^{1}$, \\ Janet R. Kumita, ${ }^{1}$ Christopher M. Dobson \& Tuomas P. J. Knowles ${ }^{1,2, *}$ \\ ${ }^{1}$ Department of Chemistry, University of Cambridge, Lensfield Road, Cambridge CB2 1EW UK \\ ${ }^{2}$ Cavendish Laboratory, University of Cambridge, JJ Thomson Avenue, Cambridge CB3 0HE, UK \\ ${ }^{\dagger}$ These authors contributed equally \\ *Corresponding author. Email tpjk2@cam.ac.uk
}

\begin{abstract}
Oligomeric intermediates are implicated as neurotoxins in the pathogenesis of protein misfolding diseases. Structural, biophysical and biochemical characterisation of these species is challenging due to their heterogeneous and transient nature, and their typically low abundance. Here, we show that microfluidic free-flow electrophoresis is capable of separating heterogeneous oligomer mixtures on a timescale of seconds, at least two orders of magnitude faster than conventional techniques. This enables analysis of oligomer structural heterogeneity, zeta-potential and immunochemistry with minimal sample perturbation under physiologicallyrelevant conditions.
\end{abstract}

\section{Introduction}

Protein misfolding is a molecular hallmark of a number of increasingly prevalent human diseases. ${ }^{1}$ Amyloid fibrils formed from misfolded proteins are the major components of the Lewy bodies and senile plaques found in the brains of patients with neurodegenerative conditions such as Parkinson's and Alzheimer's diseases, respectively. ${ }^{2}$ However, fibrillar species possess low inherent toxicity themselves, and it is instead pre-fibrillar, oligomeric aggregates that are implicated as the principal cytotoxic agents in these disorders. ${ }^{3-5}$ The characterisation and quantitation of such oligomers in the context of their toxicity and aggregation propensity is therefore an area of intense interest. ${ }^{6 ; 7}$ However, deciphering the structural attributes of oligomeric species, which 
exist as an intrinsically heterogenous population of structures during protein aggregation, is not possible using established methods that report the properties of analyte mixtures in an ensembleaveraged manner. Although measurements of oligomer populations, taken throughout protein aggregation processes, have enabled coarse-grained relationships between structure and toxicity to be defined, ${ }^{8 ; 9}$ they do not permit detailed characterisation of oligomer properties including క-potential, which may also play a crucial role in determining oligomer aggregation propensity. ${ }^{10}$ Furthermore, oligomers exist in a complex, dynamic milieu of protein-protein interactions, but current methodologies are often incapable of probing such systems on a timescale relevant to temporal changes in oligomer populations. ${ }^{11}$ Crucially, current methods for the separation of heterogeneous protein mixtures such as size exclusion chromatography (SEC), analytical ultracentrifugation (AUC) and native gel electrophoresis operate on a timescale of minutes to hours, rather than seconds as enabled by the approach shown here. In addition, existing techniques for oligomer analysis often perturb the system in question by dilution ${ }^{12}$ or use of non-solution state approaches, ${ }^{13 ; 14}$ whereas our method allows arbitrary sample conditions and solution-phase analysis.

To address these challenges, we have developed an approach based upon multi-spectral microchip free-flow electrophoresis ( $\mu \mathrm{FFE}$ ) that achieves rapid solution-phase fractionation and in-situ analysis of heterogeneous mixtures of protein oligomers and monomers. We focus on the aggregation and oligomerisation of alpha-synuclein $(\alpha S)$, a protein that is strongly implicated in the pathogenesis of Parkinson's disease. ${ }^{15}$ Using this approach, complex oligomeric mixtures are fractionated on-chip to afford rapid oligomer quantification and characterisation of ensemble heterogeneity, whilst allowing simultaneous measurement of oligomer $\zeta$-potential, a hitherto inaccessible parameter. In addition, the short experimental timescale (analysis time $\sim 5 \mathrm{~s}$ ), solution-phase conditions and minimal sample dilution enable an accurate, native-state snapshot of dynamic oligomer populations with high temporal resolution. First, we demonstrate the principle of our method through analysis of stable, fluorophore-labelled kinetically trapped $\alpha \mathrm{S}$ oligomers, ${ }^{11}$ before applying the technique to the analysis of transient oligomers that arise during protein aggregation. Moreover, by use of an oligomer-selective aptamer probe, we further demonstrate the broad applicability of our method by investigation of wild-type, unlabelled $\alpha \mathrm{S}$ oligomers.

\section{Results and Discussion}

To begin, we focus on the electrophoretic separation of monomeric $\alpha \mathrm{S}$ from a mixture of kineticallytrapped, stable, $\alpha \mathrm{S}$ oligomers. ${ }^{11}$ Our approach is based on a $\mu \mathrm{FFE}^{16 ; 17}$ platform $^{18}$ that allows rapid fractionation of samples containing a complex mixture of oligomeric and monomeric proteins (Figure 1(a)). The sample, flanked by an auxiliary buffer, is passed under laminar flow through the microfluidic chip whilst an electric field is applied perpendicular to the flow direction, resulting in fractionation of the heterogeneous mixture according to the different electrophoretic mobilities of the sample components (Figure 1(b)). Notably, in contrast to other $\mu$ FFE platforms, our device 
incorporates an in-line sample-desalting module for rapid ( $\sim 2$ s) sample preparation on chip (Figure S1, Supporting Information). This enables the use of high-salt, physiologically-relevant buffers such as PBS, which are otherwise inaccessible in $\mu \mathrm{FFE}$, as excessive ionic conduction through the buffer prevents the application of electric field. We introduce $\alpha \mathrm{S}$ monomer labelled with a fluorophore (Alexa546) orthogonal to that of the oligomeric protein mixture (Alexa488) as an in-situ reference, to allow facile in-situ differentiation between monomeric and oligomeric $\alpha \mathrm{S}$ fractions (Figure 1(b)). For this approach to be effective, it is necessary for both labelling variants to possess the same electrophoretic mobility; the dyes were chosen due to their same inherent charge, ${ }^{19}$ and quantification of the mobilities of the two labelling variants confirmed that they were identical, with $\mu=-1.43 \pm 0.11 \times 10^{-8} m^{2} V^{-1} s^{-1}$ (Figure S2).

We initially demonstrate separation of $\alpha \mathrm{S}$ monomers from kinetically-trapped, stable $\alpha \mathrm{S}$ oligomers prepared both in high and low-salt conditions (Figure 1(c)). Mixtures of kinetically stable, Alexa488-labelled oligomers together with both Alexa488 and 546-labelled monomer in PBS or $10 \mathrm{mM}$ sodium phosphate buffer were analysed, two distinct fractions were observed for the oligomeric mixture (Alexa488), but only a single fraction was present for the monomer-only sample (Alexa546). By comparison to the Alexa546 fluorescence profile, the lower and higher mobility fractions could be clearly identified as monomeric and oligomeric protein, respectively (Figure 1(d, e)). As expected, a broad peak width is observed for monomer, resulting from faster diffusion due to its smaller hydrodynamic radius in comparison to oligomeric species. This analysis revealed significant oligomer heterogeneity, with a similar electrophoretic profile for both conditions comprising a major oligomer population at $\mu=-2.49 \pm 0.16 \times 10^{-8} \mathrm{~m}^{2} V^{-1} s^{-1}$ combined with a significant high-mobility shoulder composed of smaller sub-populations. Additional data, definition of apparent mobility and further discussion of desalting- $\mu$ FFE are provided in the Supporting Information.

We attribute the electrophoretic separation of oligomer and monomer to the faster scaling of effective oligomer charge relative to oligomer size, for an oligomer of $n_{m}$ monomer units. Oligomer electrophoretic mobility $\left(\mu_{o}\right)$ is proportional to oligomer charge $\left(q_{o}\right)$ and inversely proportional to oligomer hydrodynamic radius $\left(r_{o}\right)$ according to $\mu_{o} \propto \frac{q_{o}}{r_{o}} \propto \frac{n_{m}^{\nu}}{r_{o}}$, where $\nu$ is a scaling exponent that links $q_{o}$ with $n_{m}$. For spherical oligomers, as in the case of $\alpha \mathrm{S},{ }^{11}$ we approximate that $n_{m}=\frac{V_{o}}{V_{m}}=\frac{r_{o}^{3}}{r_{m}^{3}}$ where $V_{o}, V_{m}$ and $r_{m}$ represent the oligomer and monomer volumes and monomer hydrodynamic radius, respectively. Together, these expressions yield the oligomer electrophoretic mobility as a function of $n_{m}$ according to Equation 1, where $\nu *=\nu-\frac{1}{3}$. According to this relationship, oligomers are expected to have higher mobilities than monomeric protein, and oligomer mobility is predicted to increase with oligomer size.

$$
\mu_{o} \propto \frac{n_{m}^{\nu}}{r_{m} n_{m}^{\frac{1}{3}}} \propto \frac{n_{m}^{\nu *}}{r_{m}}
$$

To verify this size-mobility relationship, we compared $\mu$ FFE to AUC, which is an established technique for generating size distributions of complex samples. A similar oligomer profile was 
consistently observed in both the AUC and $\mu \mathrm{FFE}$ analyses, indicating a scaling of oligomer electrophoretic mobility with oligomer size (Figure 1(f), additional data and rationalisation are provided in the SI). This observation shows that the electropherograms generated through $\mu \mathrm{FFE}$ correspond directly to size distributions of component species, as predicted by Equation 1, thus validating the $\mu \mathrm{FFE}$ approach as a tool for the analysis of oligomer structural heterogeneity. From the AUC analysis, values of $r_{o}$ were found to be in the range $5.8-11 \mathrm{~nm}$, in agreement with those determined previously. ${ }^{11}$ Moreover, by comparison of the $\mu \mathrm{FFE}$ and $\mathrm{AUC}$ data, it was possible to approximate a value for the scaling exponent $\nu=0.56$ (Supporting Information). $\nu$ describes the scaling of net oligomer charge with oligomer size according to $q_{o} \propto n_{m}^{\nu}$, representing a novel and previously inaccessible parameter for oligomer structural characterisation, as $\nu$ may be sensitive to variation in oligomer quaternary structure due to differences in monomer packing density or coordination, for example.

Having shown that our $\mu \mathrm{FFE}$ approach enables the fractionation of oligomeric protein mixtures, we analysed the resultant oligomer electropherograms to access the distribution of oligomer $\zeta$ potential, a fundamental parameter of nanoscale aggregates. The $\zeta$-potential describes interactions between particles, where it modulates the propensity of the system to aggregate further, and between aggregates and other biological components such as cell membranes. Previously, this parameter has been challenging to study for oligomeric protein aggregates, due to the high degree of structural heterogeneity intrinsic in oligomer samples that confounds conventional techniques, such as dynamic light scattering, for $\zeta$-potential analysis. ${ }^{20}$ Since our method allows direct observation of oligomer heterogeneity during free-solution electrophoresis, $\zeta$-potentials can be accurately assigned to individual oligomer populations rather than as a population average. Following normalisation, the monomer-only Alexa546 profile was subtracted from the Alexa488 signal to yield an electropherogram corresponding to oligomeric species only, and values for oligomer $\zeta$-potential were extracted from the reported electrophoretic mobilities (Figure 1(e), see SI). ${ }^{21}$ The most common oligomer $\zeta$-potential of $\zeta=-42.6 \pm 4.1 \mathrm{mV}$ is typical of electrostatically stable particulate systems, in agreement with previous studies that have quantified the electrostatic contribution to amyloid formation. ${ }^{22 ; 23}$ Notably, despite the relationship between surface charge and colloidal stability being of clear relevance to protein aggregation, few experimental studies have attempted to quantify $\zeta$-potential within heterogeneous oligomer populations, likely due to the challenges such an experiment would present for established techniques. ${ }^{24}$ The $\mu$ FFE method offers a facile method for quantifying this parameter in protein aggregation systems, introducing an important parameter for understanding the physical-chemical nature of aggregate species.

Following our analysis of kinetically-trapped, stable oligomers, we applied our $\mu$ FFE platform to the study of transient $\alpha \mathrm{S}$ oligomers formed during protein aggregation. Aliquots were periodically withdrawn from the shaking-initiated aggregation reaction of Alexa488-labelled $\alpha \mathrm{S}$ monomer, fibrillar components were removed by centrifugation before the samples were analysed by $\mu \mathrm{FFE}$. High-mobility fractions in the aggregation mixture corresponding to oligomeric $\alpha \mathrm{S}$ could be identified and quantified (Figure 2(a, b, c) ), following comparison of the electrophoresis profiles for the 


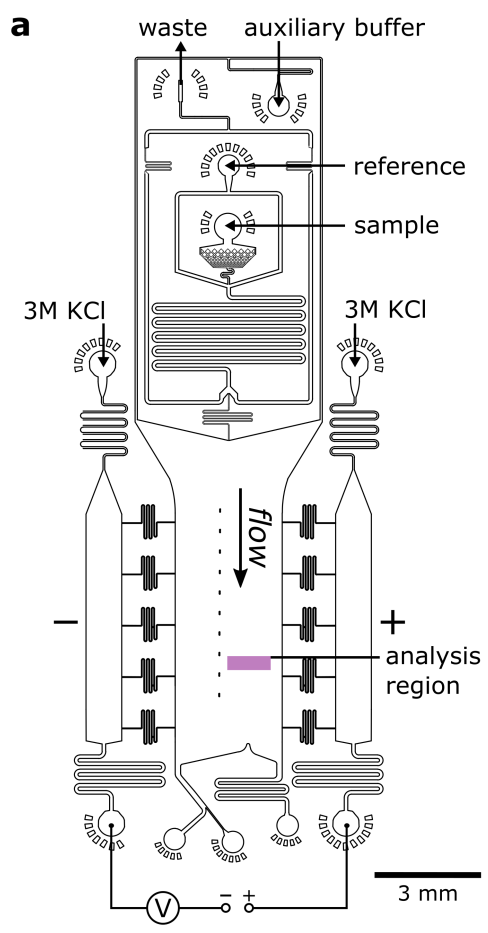

b
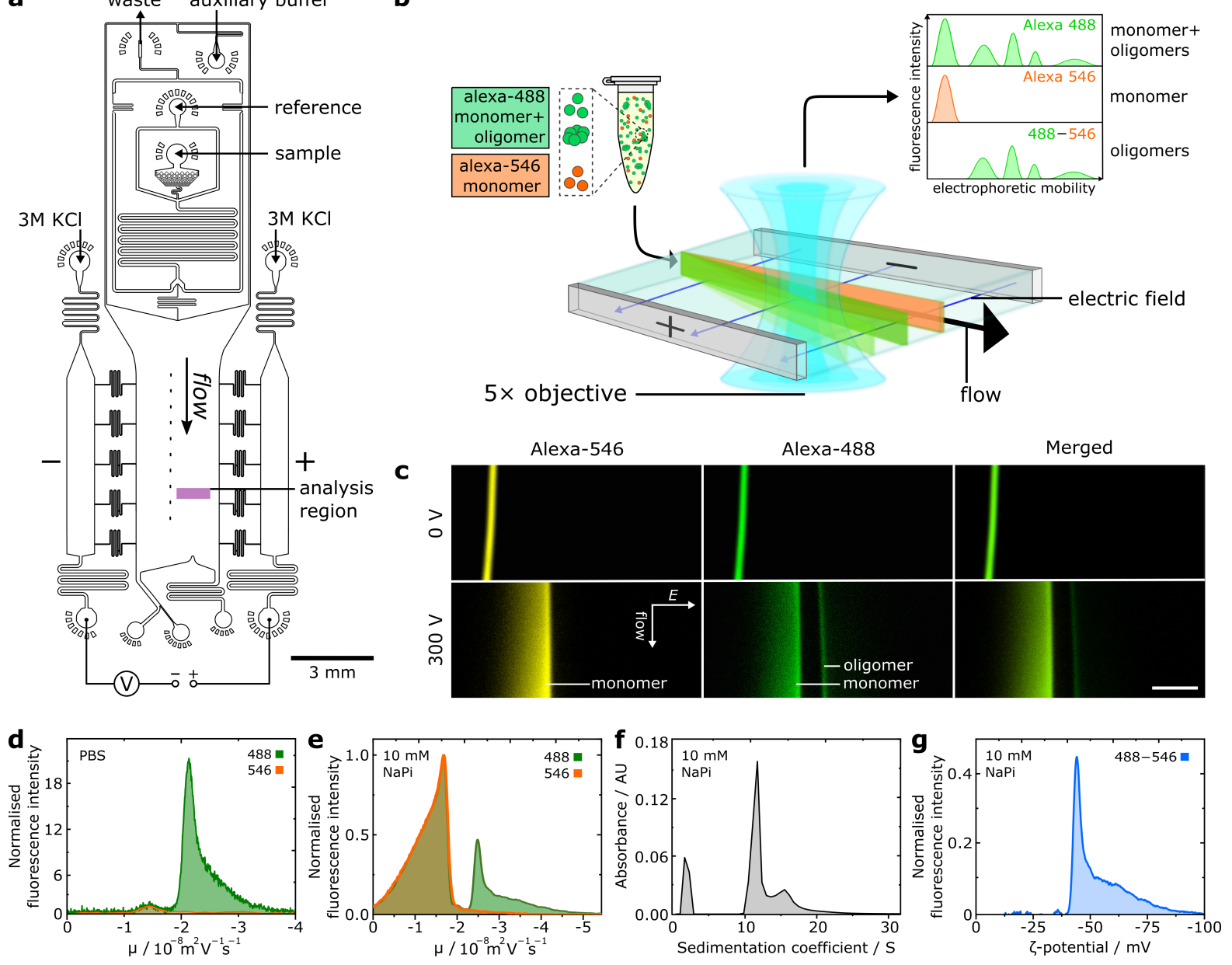

Figure 1: (a) Schematic of device design for desalting- $\mu$ FFE. The device operates through liquid $\mathrm{KCl}$ electrodes, allowing voltage application downstream of the electrophoresis chamber to prevent disruption of flow by electrolysis products. ${ }^{18}$ Reference sample can be introduced on-chip in pure water diluent, enabling rapid in-situ sample desalting prior to downstream electrophoresis. (b) Schematic for two-colour microfluidic free-flow electrophoresis of oligomeric $\alpha \mathrm{S}$. (c) Fluorescence images of Alexa546-labelled $\alpha \mathrm{S}$ monomer reference and Alexa488-labelled oligomeric mixture undergoing $\mu \mathrm{FFE}$ in $10 \mathrm{mM}$ sodium phosphate (NaPi) buffer ( $\mathrm{pH} 7.4$ ). Direction of fluid flow and electric field $(E)$ shown by arrows, scale bar $=300 \mu \mathrm{m}$. (d) Electropherograms for Alexa546 monomer reference and Alexa488-labelled $\alpha \mathrm{S}$ oligomeric mixture fluorescence in PBS buffer. (e) Electropherograms for $\alpha \mathrm{S}$ monomer and oligomeric mixture in $10 \mathrm{mM} \mathrm{NaPi}$ buffer. (f) AUC data for the same oligomer sample for data shown in $(\mathrm{e})$, the peak at $\mathrm{S} \approx 2$ is due to monomeric protein. (g) Distribution of oligomer $\zeta$-potential. 
aggregation mixture and the Alexa546-labelled monomer reference, which yielded electropherograms corresponding to oligomeric $\alpha \mathrm{S}$ alone (Figure $2(\mathrm{~d})$ ).

No oligomers were observed prior to aggregation initiation, before their concentration increased through the lag phase of the reaction, reaching a peak concentration of $1.75 \mu \mathrm{M}$ (monomer equivalent) with concomitant initiation of protein fibrillisation after $36 \mathrm{~h}$. This behaviour is in good agreement with studies employing single-molecule FRET (smFRET) experiments. ${ }^{25}$ Furthermore, two distinct oligomeric subpopulations are observable in the aggregation timecourse, which corresponds well with smFRET experiments where two major subpopulations of $\alpha \mathrm{S}$ oligomers have been observed. ${ }^{8 ; 9}$ Although our recorded values of oligomer concentration are approximately three times larger than those reported by smFRET techniques, we suggest this is because of inherent under-sampling in the smFRET approach, due to oligomer dissociation caused by sample dilution. ${ }^{12 ; 26}$ Dilution is not required for $\mu \mathrm{FFE}$, enabling accurate oligomer quantitation as evidenced by close agreement with values obtained by other techniques such as size exclusion chromatography. ${ }^{8}$ Notably, the $\mu \mathrm{FFE}$ experiment $(\approx 5 \mathrm{~s}$ analysis time $)$ is three orders of magnitude faster than methods such as AUC. This crucial feature allowed access to transient species that would be otherwise challenging to observe by AUC or other bulk separation methods, with minimal sample consumption of only a few $\mu \mathrm{L}$.

Further to the fluorophore-tagged protein systems discussed so far, $\mu \mathrm{FFE}$ can be employed for the analysis of wild-type oligomeric species when used in conjunction with an appropriate oligomerbinding probe. We demonstrate this capability by utilising a fluorophore-tagged aptamer selective for $\alpha \mathrm{S}$ oligomers. ${ }^{27}$ As shown previously, ${ }^{28}$ protein-aptamer binding retards the electrophoretic mobility of the aptamer, enabling separation of the bound and unbound fractions (Figure 3(a)). Aliquots taken from stirring-induced aggregation of wild-type $\alpha \mathrm{S}$ were incubated with the aptamer and analysed by $\mu$ FFE (Figure $3(\mathrm{~b})$ ). After $4.25 \mathrm{~h}$ aggregation time, an additional fraction at lower mobility than the unbound aptamer was observed. By peak integration, an upper bound of $80 \mathrm{nM}$ oligomer concentration could be estimated (Figure 3(c)), indicating, by comparison to labelled oligomer quantitation ((Figure 2(c)), that the aptamer may be selective for a particular subset of the overall oligomer population. The oligomeric nature of the peak was confirmed by $\mu \mathrm{FFE}$ of the aptamer in the presence of $\alpha \mathrm{S}$ monomer alone and sonicated $\alpha \mathrm{S}$ fibrils (Supporting Information). These data show that label-free, wild-type oligomers can be observed and quantified by $\mu \mathrm{FFE}$, and demonstrates the versatility of our method towards applications involving immuonchemistry-based oligomer detection and manipulation.

In conclusion, the $\mu \mathrm{FFE}$ platform presented here is a multifaceted tool for the observation and analysis of oligomeric proteins relevant to misfolding disease. $\mu \mathrm{FFE}$ affords facile, rapid analysis of structural heterogeneity within populations of transient, on-pathway oligomers formed during aggregation under native conditions, with the key advantage that unstable, weakly-interacting oligomers are not perturbed by sample dilution. Electrophoretic analysis enables electrostatic characterisation of oligomers according to their $\zeta$-potential, despite their inherent heterogeneity. Significantly, the $\mu$ FFE method also facilitates analysis of wild-type, label-free oligomers and their 

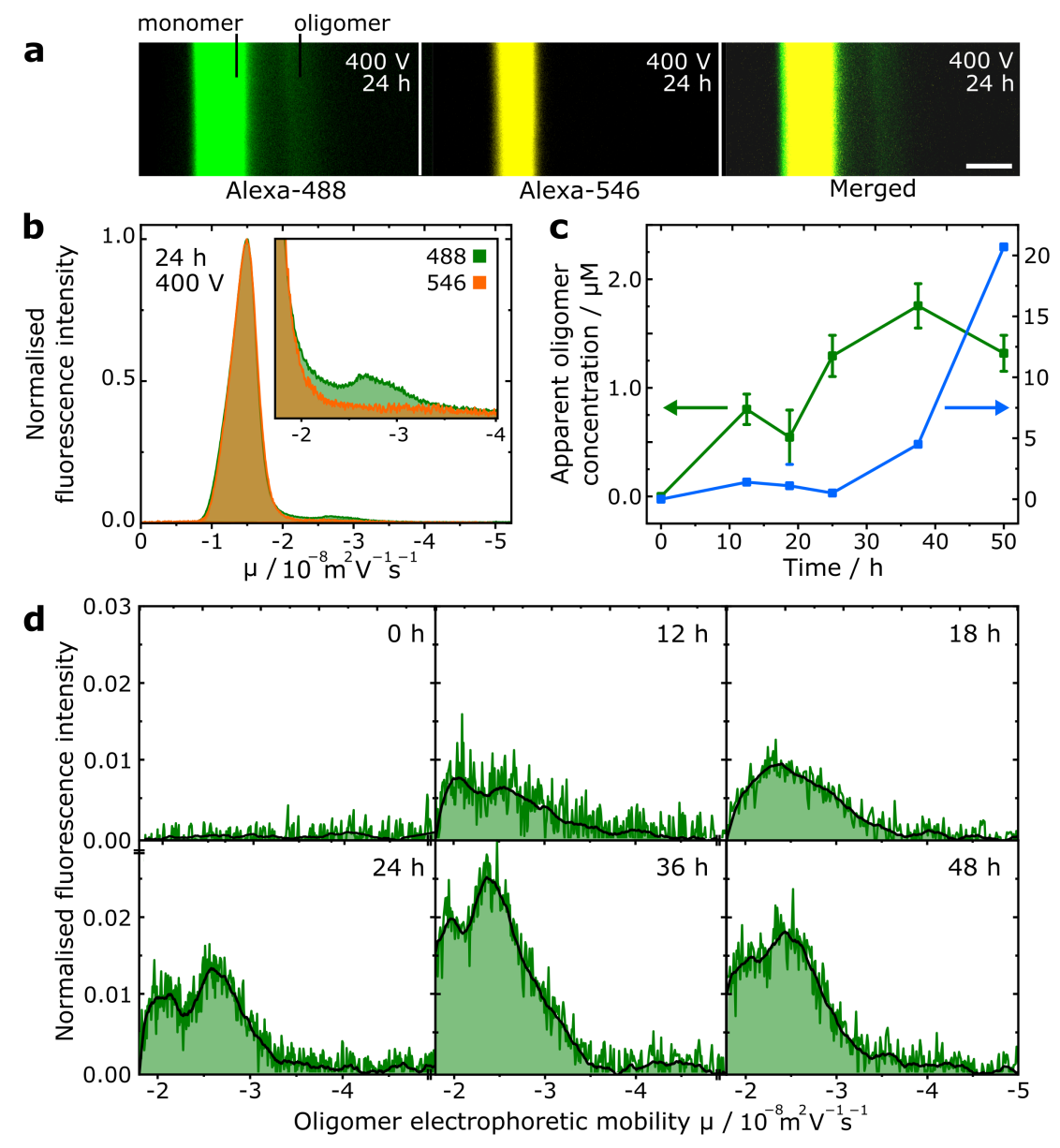

Figure 2: (a) Fluorescence images of $\mu \mathrm{FFE}$ experiment of transient oligomers formed after $24 \mathrm{~h}$ $\alpha \mathrm{S}$ aggregation. Scale bar $=300 \mu \mathrm{m}$. (b) Superimposed electropherograms of normalised alexa488 and 546 fluorescence for micrographs shown in (a), (inset) magnification of electropherograms showing oligomeric region. (c) Scatter plot showing progression of oligomer concentration (monomer equivalents) and extent of fibril conversion over the sampled time points. Error bars correspond to standard deviation of three repeat measurements of the same sample. (d) Electropherograms and smoothed traces corresponding to oligomeric species only at each sampled aggregation time point. 

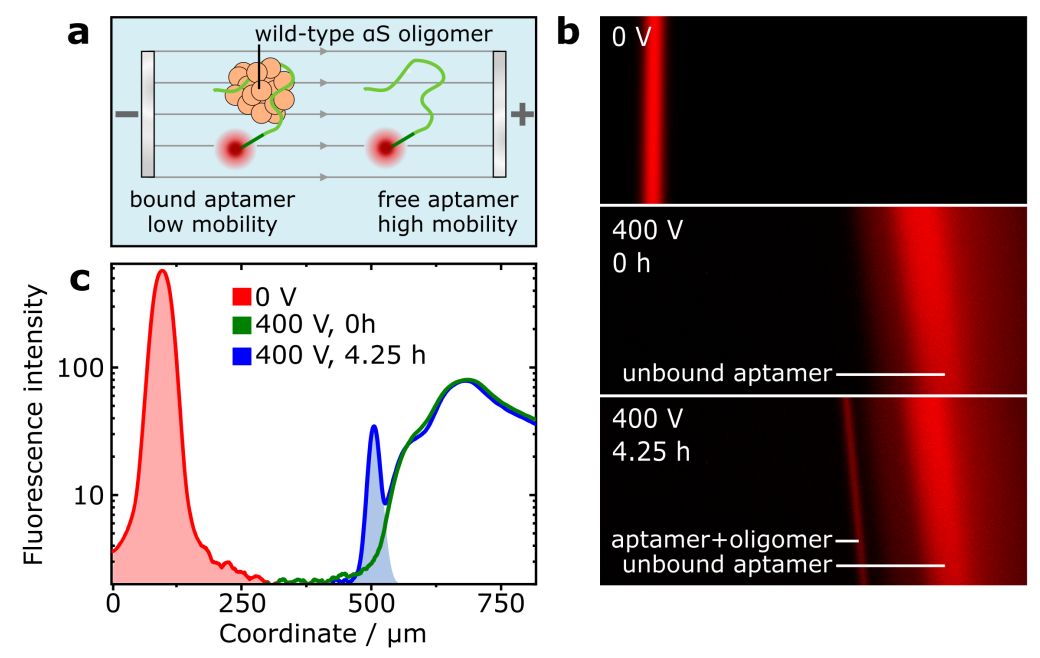

Figure 3: (a) Schematic for fractionation of oligomer-bound and unbound aptamer by $\mu F F E$. (b) Images of aptamer fluorescence during $\mu \mathrm{FFE}$, from aptamer mixed into an aggregation reaction of wild-type $\alpha \mathrm{S}$ at $0 \mathrm{~h}$ and $4.25 \mathrm{~h}$ timepoints. Additional, lower-mobility fraction after $4.25 \mathrm{~h}$ indicates aptamer-oligomer binding. (c) Analysis and integration of aptamer electropherograms enable approximation of the concentration of aptamer-bound oligomers.

interaction with immuno-reagents. Oligomer- $\mu$ FFE has many potential applications, such as in the analysis of protein aggregation kinetics, the links between oligomer structure and toxicity, and for probing protein-protein and protein-small molecule interactions relevant towards the design of therapeutic interventions. 


\section{Methods}

\section{Expression and purification of $\alpha$-synuclein}

Wild-type $\alpha$-synuclein was expressed and purified as previously described. ${ }^{29}$ Briefly, Escherichia coli BL21 cells overexpressing $\alpha$-synuclein were collected by centrifugation $\left(20 \mathrm{~min}, 4000 \mathrm{rpm}, 4{ }^{\circ} \mathrm{C}\right)$ in a JLA-8.1000 rotor in a Beckman Avanti J25 centrifuge (Beckman Coulter), resuspended in lysis buffer (10 mM Tris, $1 \mathrm{mM}$ EDTA, protease inhibitor), and lysed by sonication on ice. Following centrifugation (JA-25.5 rotor, $20 \mathrm{~min}, 18,000 \mathrm{rpm}, 4{ }^{\circ} \mathrm{C}$ ), heat-sensitive proteins were precipitated out of the lysate supernatant by boiling, and subsequently removed by centrifugation (JA-25.5 rotor, $15 \mathrm{~min}, 18,000 \mathrm{rpm}, 4{ }^{\circ} \mathrm{C}$ ). DNA was precipitated out by incubation with streptomycin sulphate $\left(10 \mathrm{mg} / \mathrm{mL}, 15 \mathrm{~min}, 4^{\circ} \mathrm{C}\right)$, and removed by centrifugation. $\alpha$-synuclein was precipitated out of the supernatant by the slow addition of ammonium sulphate $(361 \mathrm{mg} / \mathrm{mL})$ while stirring $\left(30 \mathrm{~min}, 4{ }^{\circ} \mathrm{C}\right)$. The pellet containing $\alpha$-synuclein was collected by centrifugation (JA-25.5 rotor, $15 \mathrm{~min}, 18,000 \mathrm{rpm}, 4{ }^{\circ} \mathrm{C}$ ) and resuspended in $25 \mathrm{mM}$ Tris buffer, $\mathrm{pH} 7.4,20{ }^{\circ} \mathrm{C}$. Following dialysis to ensure complete buffer exchange, the protein was loaded onto a HiLoadTM 26/10 Q Sepharose high performance column (GE Healthcare), and eluted at $\sim 350 \mathrm{mM} \mathrm{NaCl}, 20{ }^{\circ} \mathrm{C}$ with a salt gradient from $0 \mathrm{M}$ to $1.5 \mathrm{M} \mathrm{NaCl}$. Selected fractions were subsequently loaded onto a Superdex 75 26/60 (GE Healthcare) at $20{ }^{\circ} \mathrm{C}$ and eluted in PBS, pH 7.4, $20{ }^{\circ} \mathrm{C}$. Protein concentration was determined by absorbance at $275 \mathrm{~nm}$, using an extinction coefficient of $5600 \mathrm{M}^{-1} \mathrm{~cm}^{-1}$. The cysteine-containing variant $(\mathrm{N} 122 \mathrm{C}$ ) of $\alpha$-synuclein was purified by the same protocol, with the addition of $3 \mathrm{mM}$ DTT to all buffers.

\section{Labelling of $\alpha$-synuclein}

$\alpha$-Synuclein protein was fluorophore-labelled to enable visualisation by fluorescence microscopy. ${ }^{11}$ In order to remove DTT, cysteine variants of $\alpha$-synuclein were buffer exchanged into PBS or sodium phosphate buffer by use of P10 desalting columns packed with Sephadex G25 matrix (GE Healthcare). The protein was then incubated with an excess of Alexa-488 or Alexa-546 dyes with maleimide moieties (Thermofisher Scientific) (overnight, $4{ }^{\circ} \mathrm{C}$ on a rolling system) at a molar ratio of 1:1.5 (protein-to-dye). The labelling mixture was loaded onto a Superdex 200 16/600 (GE Healthcare) and eluted in PBS or $10 \mathrm{mM}$ sodium phosphate $\mathrm{pH} 7.4$ buffer at $20{ }^{\circ} \mathrm{C}$, to separate the labelled protein from free dye. The concentration of the labelled protein was estimated by the absorbance of the fluorophores, assuming a 1:1 labelling stoichiometry (Alexa-488: $72000 \mathrm{M}^{-1}$ $\mathrm{cm}^{-1}$ at $495 \mathrm{~nm}$, Alexa-546: $112000 \mathrm{M}^{-1} \mathrm{~cm}^{-1}$ at $\left.556 \mathrm{~nm}\right)$.

\section{Preparation of stable $\alpha$-synuclein oligomers}

$\alpha$-synuclein oligomers were prepared by the following procedure. ${ }^{11}$ Monomeric $\alpha$-synuclein was lyophilised in Milli-Q water and subsequently resuspended in PBS or $10 \mathrm{mM}$ sodium phosphate 
buffer, both at $\mathrm{pH} 7.4$, to give a final concentration of ca. $840 \mathrm{uM}(12 \mathrm{mg} / \mathrm{mL})$. The resulting solution was passed through a $0.22 \mathrm{uM}$ cut-off filter before incubation at $38{ }^{\circ} \mathrm{C}$ for $20-24 \mathrm{~h}$ under quiescent conditions. Very small amounts of fibrillar species formed during this time were removed by ultracentrifugation for $1 \mathrm{~h}$ at $90000 \mathrm{rpm}$ (Optima TLX Ultracentrifuge, Beckman Coulter, TLA-120.2 Beckman rotor, $288000 \mathrm{~g}$ ). The excess monomeric protein and some small oligomers were then removed by multiple filtration steps using $100-\mathrm{kDa}$ cut-off membranes. The final concentration of oligomers was estimated on the basis of the absorbance at $495 \mathrm{~nm}$ using a molar extinction coefficient of $72000 \mathrm{M}^{-1} \mathrm{~cm}^{-1}$ for the Alexa-488 labelled oligomers.

\section{Procedure for generation and isolation of kinetic $\alpha$-synuclein oligomers}

Alexa-488 labelled $\alpha$-synuclein (N122C, $100 \mu \mathrm{M}, 500-800 \mu \mathrm{L}$ ) was incubated in PBS buffer, pH 7.4 , at $37{ }^{\circ} \mathrm{C}$ with $0.01 \%$ sodium azide, in a $1.5 \mathrm{~mL}$ Eppendorf tube with shaking at $200 \mathrm{rpm}$ over 96 hours. $150 \mu \mathrm{L}$ aliquots were withdrawn and centrifuged for $15 \mathrm{~min}$ at $21130 \mathrm{~g}$, to pellet insoluble, fibrillar components of the reaction mixture. The supernatant, containing monomeric and oligomeric $\alpha$-synuclein, was carefully removed and used immediately for FFE experiments. A small portion of the supernatant was retained for analysis of $\alpha$-synuclein concentration by UV-vis absorption spectroscopy.

\section{Microfluidic device fabrication}

Devices were designed using AutoCAD software (Autodesk) and photolithographic masks printed on acetate transparencies (Micro Lithography Services). Polydimethylsiloxane (PDMS) devices were produced on SU-8 moulds fabricated via photolithographic processes as described elsewhere, ${ }^{30 ; 31}$ with UV exposure performed with custom-built LED-based apparatus. ${ }^{32}$ Following development of the moulds, feature heights were verified by profilometer (Dektak, Bruker) and PDMS (Dow Corning, primer and base mixed in 1:10 ratio) applied and degassed before baking at $65{ }^{\circ} \mathrm{C}$ overnight. Devices were cut from the moulds and holes for tubing connection $(0.75 \mathrm{~mm})$ and electrode insertion $(1.5 \mathrm{~mm})$ were created with biopsy punches, the devices were cleaned by application of Scotch tape and sonication in IPA (5 min). After oven drying, devices were bonded to glass slides using an oxygen plasma. Before use, devices were rendered hydrophilic via prolonged exposure to oxygen plasma. ${ }^{33}$

\section{$\mu$ FFE device operation}

Liquid-electrode $\mu \mathrm{FFE}$ devices were operated as described previously. ${ }^{18}$ Briefly, fluids were introduced to the device by PTFE tubing, 0.012"ID x 0.030"OD (Cole-Parmer) from glass syringes (Gas Tight, Hamilton) driven by syringe pumps (Cetoni neMESYS). $\mu$ FFE experiments were conducted with auxiliary buffer, electrolyte, monomer reference and sample flow rates of 1200, 250, 
140 and $10 \mu \mathrm{L} \mathrm{hr}{ }^{-1}$, respectively, for $15 \times$ reduction in buffer salt concentration for samples in PBS buffer.

Potentials were applied by a programmable benchtop power supply (Elektro-Automatik EA-PS 9500-06) via bent syringe tips inserted into the electrolyte outlets. Device voltage efficiency was calibrated by comparison of current-voltage curves of the device operating under assay conditions and when filled with $3 \mathrm{M} \mathrm{KCl}$ electrolyte. Efficiencies were found to be $\approx 20 \%$, affording electric fields equivalent to $200-267 \mathrm{Vcm}^{-1}$ for potentials of $300-400 \mathrm{~V}$.

Microfluidic experiments were conducted using an inverted fluorescence microscope (Zeiss AxioObserver D1), Alexa488, 546 and 647-labelled species were observed using appropriate filter sets (49002, 49004 and 49006, Chroma Technology) and camera (Evolve 512 CCD, Photometrics).

\section{Oligomer $\mu$ FFE experiments}

Alexa488-labelled oligomeric mixtures (4 $\mu \mathrm{M}$ monomer equivalent for stabilised oligomers) in either 10 mM sodium phosphate pH 7.4 or PBS buffer were mixed on-chip with Alexa546-labelled monomer $(2 \mu \mathrm{M})$ in either $10 \mathrm{mM}$ sodium phosphate $\mathrm{pH} 7.4$ or pure water, respectively. For oligomeric samples in $10 \mathrm{mM}$ sodium phosphate or PBS buffer, auxiliary buffer comprised of the same or 15X diluted PBS buffer, respectively, supplemented with $0.05 \% \mathrm{v} / \mathrm{v}$ Tween-20.

Following data acquisition, Alexa488 and Alexa546 fluorescence profiles were extracted, superimposed and normalised to the peak fluorescence of the Alexa546 monomer. Subtraction of the normalised Alexa546 from the Alexa488 profile afforded profiles due to oligomeric aggregates alone. For quantification of oligomers formed transiently during $\alpha \mathrm{S}$ aggregation, the relative peak integrals of oligomeric and monomer populations were compared to the known monomer concentration and degree of sample loss during desalting (see Supporting Information) to calculate the oligomer concentration.

\section{Aptamer-oligomer $\mu$ FFE experiments}

Wild-type $\alpha \mathrm{S}(100 \mu \mathrm{M})$ was mixed with Alexa647-labelled aptamer $(2 \mu \mathrm{M}$, Integrated DNA Technologies, sequence is provided in the Supporting Information) in PBS buffer and underwent aggregation by rapid stirring. ${ }^{34} 150 \mathrm{uL}$ aliquots were withdrawn every hour and fibrillar material was removed by centrifugation. The sample then underwent desalting- $\mu$ FFE analysis as described above.

\section{Analytical ultra-centrifugation}

Sedimentation velocity measurements ${ }^{35}$ were carried out at $20{ }^{\circ} \mathrm{C}, 43000 \mathrm{rpm}$ (136680 g), using a Beckman Coulter Optima XL-1 analytical ultracentrifuge equipped with UV-visible absorbance 
optics and an AN50-Ti rotor. The sedimentation coefficient distributions, corrected to standard conditions by using the SEDNTERP programme, were calculated via least-squares boundary modelling of sedimentation velocity data using the $\mathrm{c}(\mathrm{s})$ and $\mathrm{ls}-\mathrm{g} *(\mathrm{~s})$ methods, as implemented in the SEDFIT programme. ${ }^{36}$

\section{Author Contributions}

W.E.A, C.K.X, J.R.K, C.M.D, T.P.J.K designed research, W.E.A, C.K.X performed research, M.C.C, T.W.H, K.L.S contributed reagents/analytic tools, W.E.A, C.K.X analyzed data and W.E.A, C.K.X, J.R.K, T.P.J.K wrote the paper.

\section{Acknowledgment}

The research leading to these results has received funding from the European Research Council under the European Union's Seventh Framework Programme (FP7/2007-2013) through the ERC grant PhysProt (agreement 337969) and from the Newman Foundation. W. E. A. acknowledges support from the EPSRC Cambridge NanoDTC, EP/L015978/1. C.K.X acknowledges support from a Herchel Smith Research Studentship. T.W.H. acknowledges support from the Oppenheimer Fund and the BBSRC. K.L.S. acknowledges support from the EPSRC. All authors are supported by the Centre for Misfolding Diseases.

\section{References}

[1] Chiti, F.; Dobson, C. M. Annual Review of Biochemistry 2017, 86, 27-68.

[2] Masters, C. L.; Simms, G.; Weinman, N. A.; Multhaup, G.; McDonald, B. L.; Beyreuther, K. Proceedings of the National Academy of Sciences 1985, 82, 4245-4249.

[3] Haass, C.; Selkoe, D. J. Nature Reviews Molecular Cell Biology 2007, 8, 101-112.

[4] Benilova, I.; Karran, E.; De Strooper, B. Nature Neuroscience 2012, 15, 349-357.

[5] Ingelsson, M. Frontiers in Neuroscience 2016, 10, 408.

[6] Bengoa-Vergniory, N.; Roberts, R. F.; Wade-Martins, R.; Alegre-Abarrategui, J. Acta neuropathologica 2017, 134, 819-838.

[7] Ono, K. Neurochemical Research 2017, 42, 3362-3371. 
[8] Cremades, N.; Cohen, S. I. A.; Deas, E.; Abramov, A. Y.; Chen, A. Y.; Orte, A.; Sandal, M.; Clarke, R. W.; Dunne, P.; Aprile, F. A.; Bertoncini, C. W.; Wood, N. W.; Knowles, T. P. J.; Dobson, C. M.; Klenerman, D. Cell 2012, 149, 1048-59.

[9] Iljina, M.; Garcia, G. A.; Horrocks, M. H.; Tosatto, L.; Choi, M. L.; Ganzinger, K. A.; Abramov, A. Y.; Gandhi, S.; Wood, N. W.; Cremades, N.; Dobson, C. M.; Knowles, T. P. J.; Klenerman, D. Proceedings of the National Academy of Sciences of the United States of America 2016, 113, E1206-15.

[10] Li, X.; Dong, C.; Hoffmann, M.; Garen, C. R.; Cortez, L. M.; Petersen, N. O.; Woodside, M. T. Scientific Reports 2019, 9, 1734.

[11] Chen, S. W. et al. Proceedings of the National Academy of Sciences of the United States of America 2015, 112, E1994-2003.

[12] Whiten, D. R.; Cox, D.; Horrocks, M. H.; Taylor, C. G.; De, S.; Flagmeier, P.; Tosatto, L.; Kumita, J. R.; Ecroyd, H.; Dobson, C. M.; Klenerman, D.; Wilson, M. R. Cell Reports 2018, 23, 3492-3500.

[13] Simone Ruggeri, F.; Habchi, J.; Cerreta, A.; Dietler, G. Current Pharmaceutical Design 2016, 22, 3950-3970.

[14] Pieri, L.; Madiona, K.; Melki, R. Scientific Reports 2016, 6, 24526.

[15] Stefanis, L. Cold Spring Harb Perspect Med 2012, 4, a009399.

[16] Turgeon, R. T.; Bowser, M. T. Anal. Bioanal. Chem. 2009, 394, 187-198.

[17] Herling, T. W.; Arosio, P.; Müller, T.; Linse, S.; Knowles, T. P. J. Physical Chemistry Chemical Physics 2015, 17, 12161-12167.

[18] Saar, K. L.; Zhang, Y.; Muller, T.; Challa, P. K.; Devenish, S.; Andrew, L.; Knowles, T. P. J. Lab Chip 2018, 18, 162-170.

[19] Zanetti-Domingues, L. C.; Tynan, C. J.; Rolfe, D. J.; Clarke, D. T.; Martin-Fernandez, M. PLOS ONE 2013, 8 .

[20] Arosio, P.; Müller, T.; Rajah, L.; Yates, E. V.; Aprile, F. A.; Zhang, Y.; Cohen, S. I. A.; White, D. A.; Herling, T. W.; De Genst, E. J.; Linse, S.; Vendruscolo, M.; Dobson, C. M.; Knowles, T. P. J. ACS Nano 2016, 10, 333-341.

[21] Doane, T. L.; Chuang, C.-H.; Hill, R. J.; Burda, C. Accounts of Chemical Research 2011, 45, 317-326.

[22] Xu, S. Amyloid 2007, 14, 119-131.

[23] Xu, S. The Journal of Physical Chemistry B 2009, 113, 12447-12455. 
[24] Stetefeld, J.; McKenna, S. A.; Patel, T. R. Biophysical reviews 2016, 8, 409-427.

[25] Iljina, M. et al. BMC Biology 2017, 15, 57.

[26] Kjaergaard, M.; Dear, A. J.; Kundel, F.; Qamar, S.; Meisl, G.; Knowles, T. P.; Klenerman, D. ACS Chemical Neuroscience 2018, 9, 3060-3071.

[27] Tsukakoshi, K.; Abe, K.; Sode, K.; Ikebukuro, K. Analytical Chemistry 2012, 84, 5542-5547.

[28] Arter, W. E.; Charmet, J.; Kong, J.; Saar, K. L.; Herling, T. W.; Müller, T.; Keyser, U. F.; Knowles, T. P. Analytical Chemistry 2018, 90, 10302-10310.

[29] Hoyer, W.; Antony, T.; Cherny, D.; Heim, G.; Jovin, T. M.; Subramaniam, V. Journal of Molecular Biology 2002, 322, 383-393.

[30] Mazutis, L.; Gilbert, J.; Ung, W. L.; Weitz, D. A.; Griffiths, A. D.; Heyman, J. A. Nat. Protocols 2013, 8, 870-891.

[31] McDonald, J. C.; Duffy, D. C.; Anderson, J. R.; Chiu, D. T.; Wu, H.; Schueller, O. J. A.; Whitesides, G. M. Electrophoresis 2000, 21, 27-40.

[32] Challa, P. K.; Kartanas, T.; Charmet, J.; Knowles, T. P. J. Biomicrofluidics 2017, 11, 014113.

[33] Tan, S. H.; Nguyen, N.-T.; Chua, Y. C.; Kang, T. G. Biomicrofluidics 2010, 4, 32204.

[34] Buell, A. K.; Galvagnion, C.; Gaspar, R.; Sparr, E.; Vendruscolo, M.; Knowles, T. P.; Linse, S.; Dobson, C. M. Proceedings of the National Academy of Sciences of the United States of America 2014, 111, 7671-7676.

[35] Harding, S. E.; Rowe, A. J.; Horton, J. C. Analytical Ultracentrifugation in Biochemistry and Polymer Science; 1992.

[36] Schuck, P. www.analyticalultracentrifugation.com/default.htm. 